\title{
Studies of Active Galaxies in Byurakan: Recent Results
}

\author{
A. M. Mickaelian, H. V. Abrahamyan, G. M. Paronyan, G. A. Mikayelyan, and M. V. \\ Gyulzadyan
}

NAS RA Byurakan Astrophysical Observatory (BAO), Byurakan 0213, Aragatzotn Province, Armenia

\begin{abstract}
We present the recent results of studies on active galaxies (both AGN and Starbursts) by the Extragalactic group of Byurakan Astrophysical Observatory (BAO) Research Department "Astronomical Surveys". The research has been carried out in 2017-2019 and the results are published in 2018-2019. These studies are characterized by multiwavelength approach to statistical analysis of large amount of data obtained in different wavelengths; from X-ray to radio. Results on HRC/BHRC sample objects (optical identifications of ROSAT X-ray sources), studies of Markarian galaxies in UV and multiwavelength SEDs, abundance and star formation determinations in Mrk galaxies from SDSS spectra, revised optical classification of "LINERs", study and classification of SDSS spectra for Byurakan-IRAS Galaxies, summary of observations and study of Byurakan-IRAS Galaxies (BIG objects), discovery of new bright ULIRGs from the IRAS PSC/FSC Combined Catalogue and their spectral classification, radio variable sources at $1400 \mathrm{MHz}$ and their optical variability, classification of BZCAT objects having uncertain types (BZU objects), and optical variability of blazars are presented.
\end{abstract}

Keywords: active galaxies, AGN, Starburst Galaxies, quasars, Seyfert galaxies, LINERs, composite spectrum objects, HII, IRAS galaxies, variable sources

\section{Introduction}

Recent results of the Extragalactic group of BAO Research Department "Astronomical Surveys" are related to multiwavelength studies of active galaxies using large amount of data from X-ray, UV, optical, IR and radio ranges, namely large-area or all-sky surveys, with heavy use of cross-correlations, classifications on activity types using our observations and SDSS spectra, building diagnostic diagrams, Spectral Energy Distributions (SEDs), etc. For classification of SDSS spectra, we have used our new approach that is focused on detailed analysis of the most important emission lines and introducing fine details, like subtypes for the main broad-line Seyfert galaxies and narrow-line Seyfert ones. Results on HRC/BHRC sample objects (optical identifications of ROSAT X-ray sources), studies of Markarian galaxies in UV and multiwavelength SEDs, abundance and star formation determinations in Mrk galaxies from SDSS spectra (for spectra having higher signal-to noise ratio), revised optical classification of "LINERs", study and classification of SDSS spectra for Byurakan-IRAS Galaxies (BIG objects), summary of observations and study of BIG objects, discovery of new bright ULIRGs from the IRAS PSC/FSC Combined Catalogue (complied earlier by our group) and their spectral classification using SDSS spectra, 6300 radio variable sources at $1400 \mathrm{MHz}$ and their optical variability, classification of BZCAT objects having uncertain types (BZU objects), and optical variability of blazars are given in individual sections. These studies have been carried out during 2017-2019 and the results are published in a number of papers in 2018-2019. Previous results can be found in the review papers by Mickaelian (2017) and by Mickaelian et al. (2017).

\footnotetext{
*aregmick@yahoo.com, Corresponding author
} 


\section{Activity Types of Galaxies Selected from HRC/BHRC Sample (Paronyan et al. 2018, 2019a,b)}

In this study we carry out detailed spectral classification of $371(173+198)$ AGN candidates from the Joint HRC/BHRC sample, which is a combination of HRC (Hamburg-ROSAT Catalogue) and BHRC (Byurakan-Hamburg-ROSAT Catalogue). These objects were revealed as optical counterparts for ROSAT X-ray sources; however, spectra for 371 of them are given in SDSS without definite spectral classification. We studied these 371 objects using the SDSS spectra and revealed the detailed activity types for them. Three diagnostic diagrams and direct examination of the spectra were used to obtain more confident classification. We also identified these sources in other wavelength ranges and calculated some of their parameters. In Figure 1 we give examples of SDSS DR15 spectra for some classified HRC-BHRC objects.

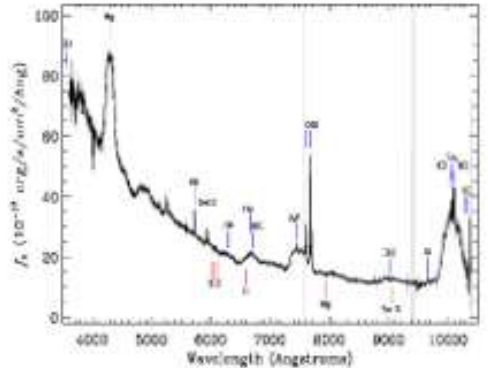

a) QSO

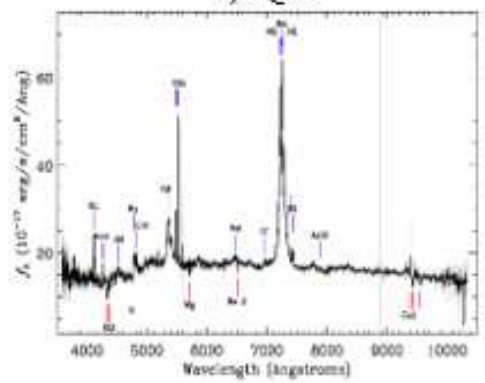

d) S1.5

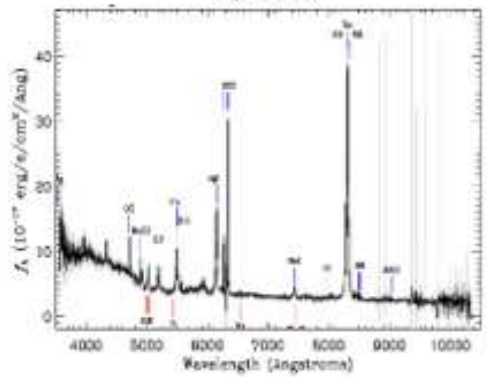

g) S2.0

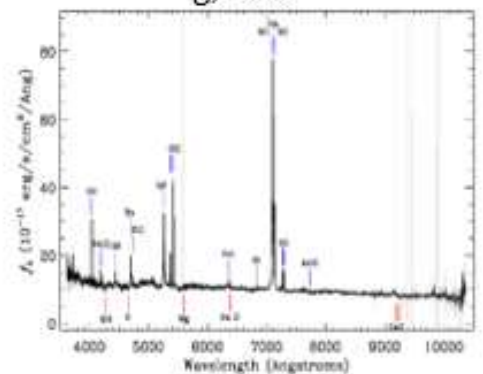

j) $\mathrm{HII}$

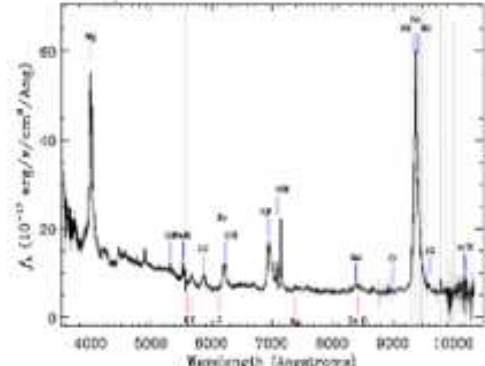

b) $\mathrm{S} 1.0$

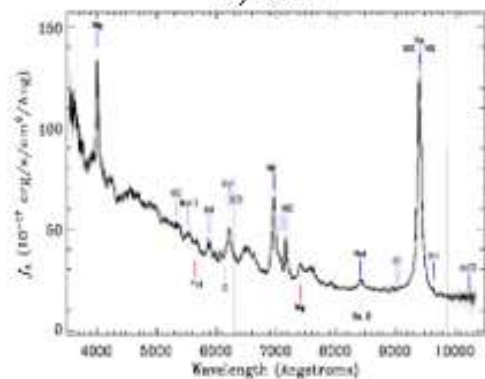

e) NLS1.5

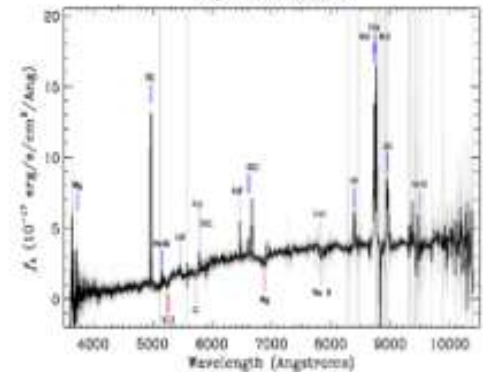

h) LINER

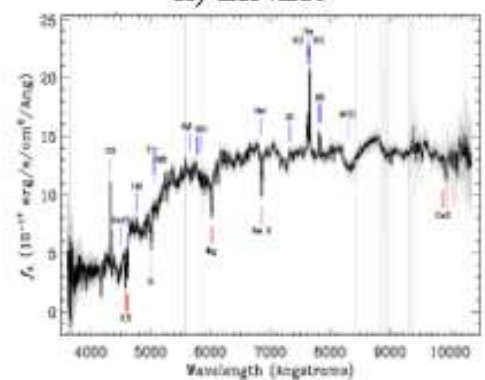

k) $\mathrm{Em}$

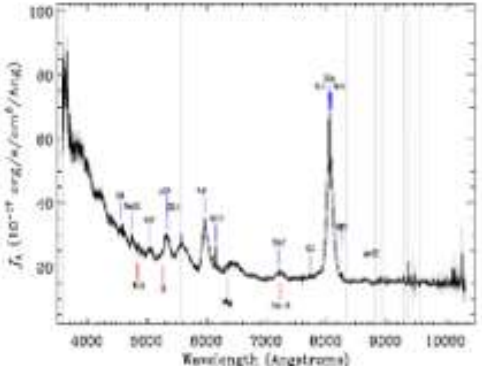

c) NLS1.0

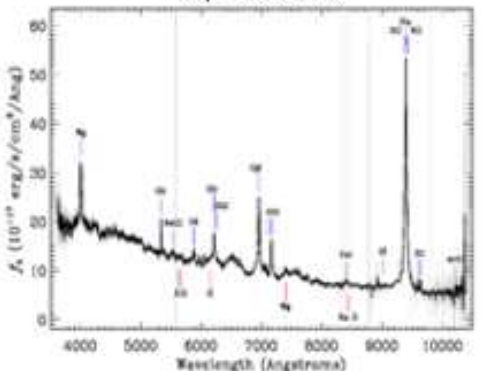

f) NLS1.8

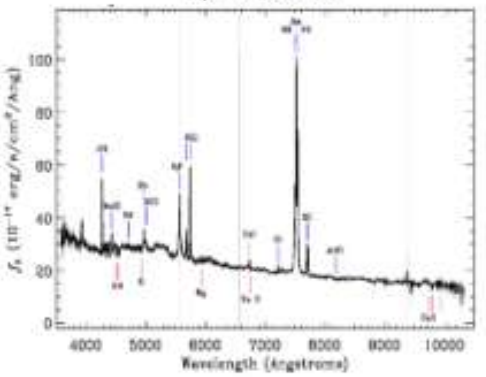

i) Composite (HII/NLS1.8)

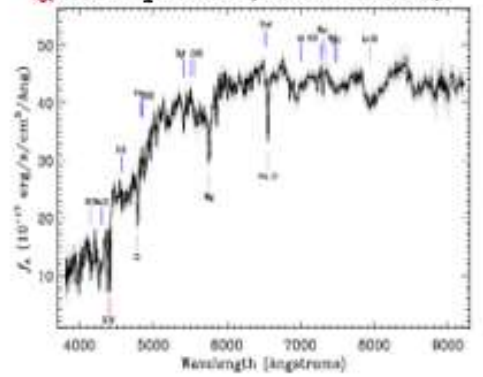

1) $\mathrm{Abs}$

Figure 1. Examples of SDSS DR15 spectra for some HRC-BHRC objects. a) QSO, b) S1.0, c) NLS1.0, d) S1.5, e) NLS1.5, f) NLS1.8, g) S2.0, h) LINER, i) HII/NLS1.8, j) HII, k) Em, l) Abs. 


\section{Markarian galaxies in UV and multiwavelength studies (Mickaelian et al. 2018a)}

The UV properties of 1152 Markarian galaxies have been investigated based on GALEX data. These objects have been investigated also in other available wavelengths using multi-wavelength data from X-ray to radio. Using our classification for activity types for 779 Markarian galaxies based on SDSS spectroscopy, we have investigated these objects on the GALEX, 2MASS and WISE colormagnitude and color-color diagrams by the location of objects of different activity types and have revealed a number of loci. UV contours overplotted on the optical images revealed additional structures, particularly spiral arms of a number of Markarian galaxies. UV (FUV and NUV) and optical absolute magnitudes and luminosities have been calculated showing graduate transition from AGN to Composites, HIIs and Absorption line galaxies from (average M) $-17.56^{m}$ to $-15.20^{m}$ in FUV, from $-18.07^{m}$ to $-15.71^{m}$ in NUV and from AGN to Composites, Absorption line galaxies and HII from $-21.14^{m}$ to $-19.42^{m}$ in optical wavelengths and from (average L) $7 \times 10^{9}$ to $4 \times 10^{8} \mathrm{~L}_{\odot}$ in FUV, from $1 \times 10^{10}$ to $5 \times 10^{8} \mathrm{~L}_{\odot}$ in NUV and from AGN to Composites, Absorption line galaxies and HII from $7 \times 10^{10}$ to $1 \times 10^{10}$ in optical wavelengths. We give in Figure 2 the distribution of GALEX FUV and NUV magnitudes by redshifts for 1152 Markarian galaxies.
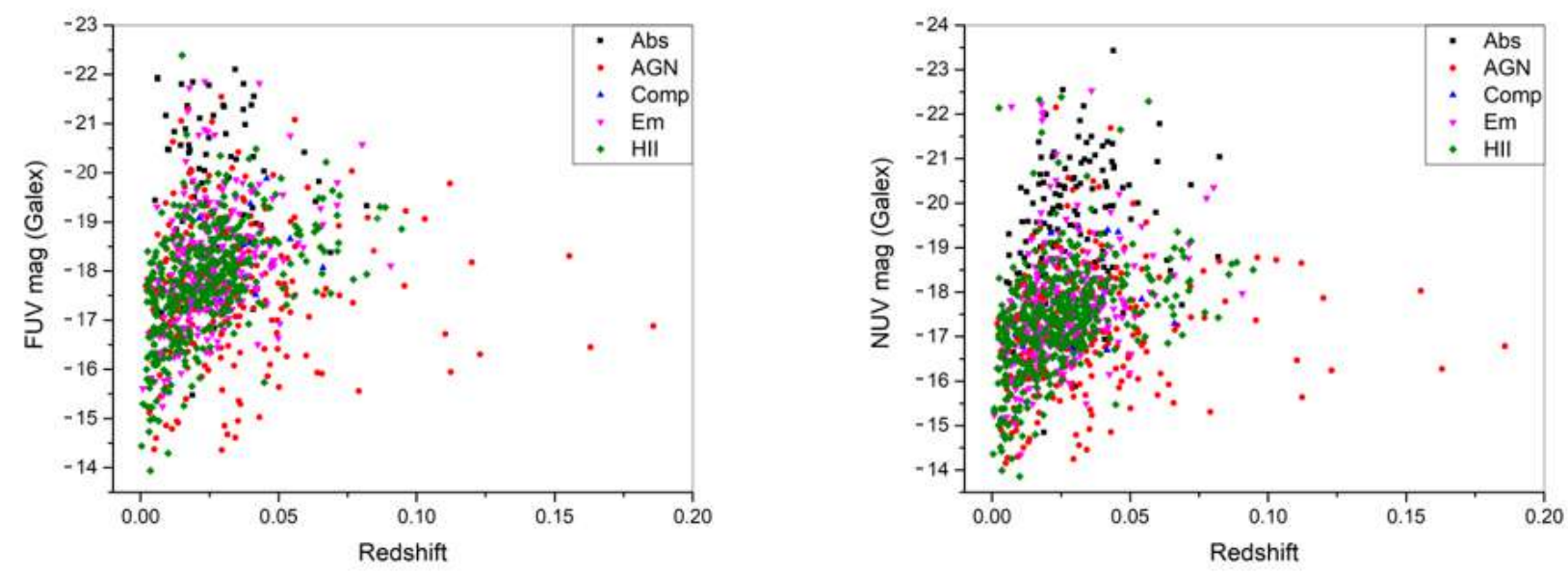

Figure 2. The distribution of GALEX FUV and NUV magnitudes by redshifts for Markarian galaxies. As expected, AGNs and Composites are detected at higher redshifts due to their higher luminosities.

\section{Abundance and Star Formation Determinations in Mrk galaxies from SDSS Spectra (Gyulzadyan et al. 2018)}

We analyze the oxygen and nitrogen abundance and specific star formation rates (sSFR) in Markarian galaxies from SDSS spectra. The Data Release 7 (DR7) of SDSS contains photometric data for more there 1000 and spectral information for more than 700 Markarian objects. The Mrk sample has played a central role in the task of distinguishing between the astrophysical different types of phenomena that occur in AGNs. In the course of the Markarian survey, more than 200 Seyfert galaxies, and hundreds of starbursts, blue compact, and H II galaxies were discovered. The Markarian survey remains perhaps the best-known source of such objects in the local universe. We have measured their line fluxes and derived the $\mathrm{O}$ and $\mathrm{N}$ abundances using recent calibrations. We have compared the oxygen and nitrogen abundances derived from global emission-line Sloan Digital Sky Survey (SDSS) spectra of galaxies using (1) the Te method and (2) two recent strong-line calibrations: the ON (Oxygen-Nitrogen) and NS (Nitrogen-Sulfur) calibrations. The behaviour of the $[\mathrm{N} / \mathrm{H}]$ ratio in under abundant regions gives strong support to a partially primary origin of nitrogen. The star formation rate (SFR) is one of the main parameters used to analyze the evolution of galaxies through time. In the local Universe, the $\mathrm{H} \alpha$ luminosity derived from IFS (Integral Field Spectroscopy) observations can 
be used to measure SFR, at least in statistically significant, optically-selected galaxy samples, once stellar continuum absorption and dust attenuation effects are accounted for.

\section{Revised optical classification of "LINERs" (Abrahamyan et al. 2018a)}

This work is dedicated to reclassification of LINERs. For our investigation we use the catalogue Véron-Cetty \& Véron 13th edition. In this catalogue 926 LINERs are included. Cross-correlation of these sources with SDSS DR14 gives 176 objects which have spectra in SDSS. Having mediumresolution spectra from SDSS we have done reclassification of these sources. As a result, $54 \%$ of these sources have changed their classification. We give in Figure 3 the dependence of absolute magnitude vs. redshift for LINERs.

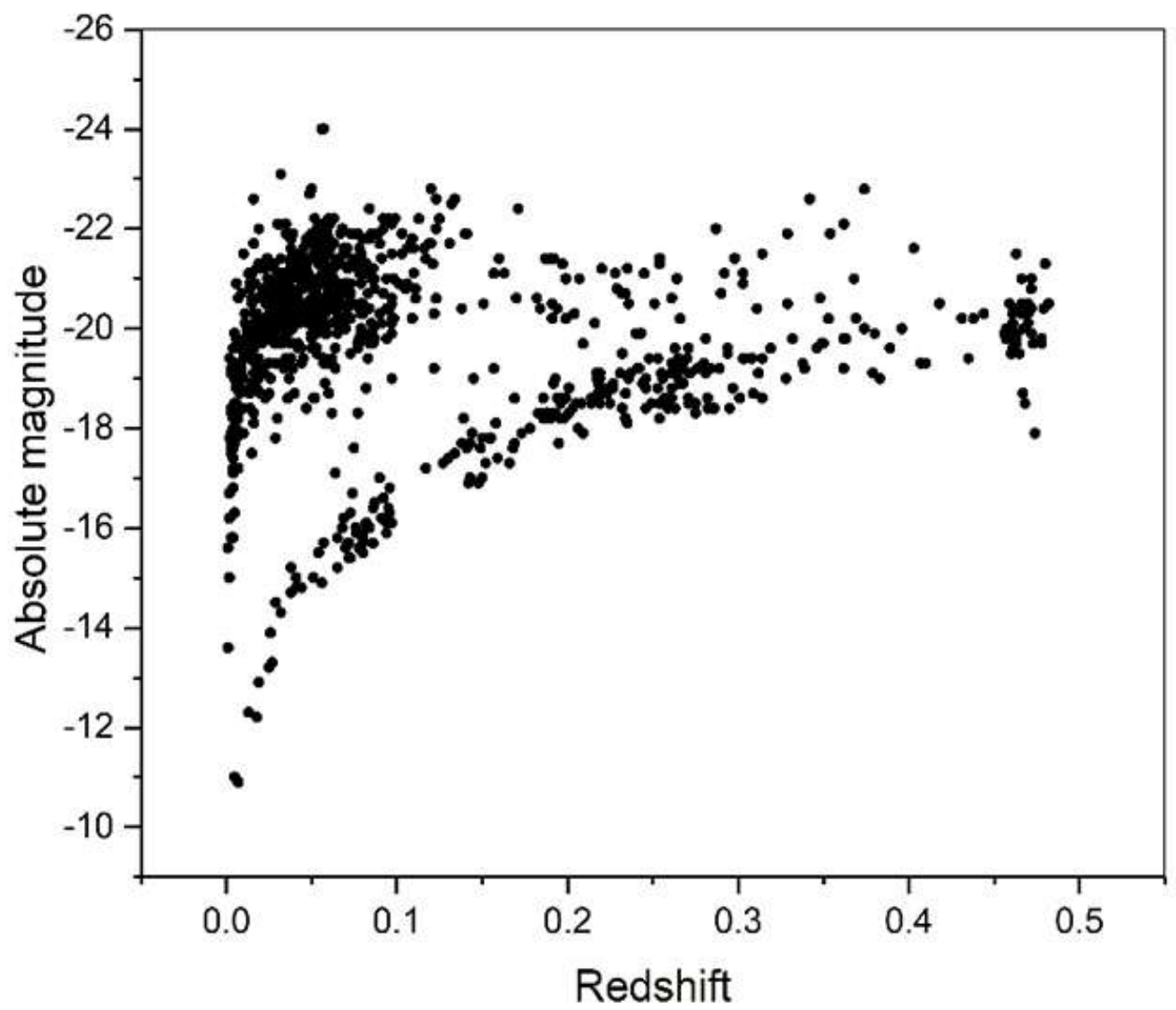

Figure 3. Absolute magnitude vs. redshift for LINERs.

\section{Study and Classification of SDSS Spectra for Byurakan - IRAS Galax- ies (Mickaelian et al. 2018b)}

The sample of Byurakan-IRAS galaxies (BIG) has been created based on optical identifications of IRAS Point Source Catalog (PSC) at high galactic latitudes. As a result, 1178 galaxies have been identified. 172 of them have been observed spectroscopically with Byurakan Astrophysical Observatory (BAO, Armenia) $2.6 \mathrm{~m}$, Special Astrophysical Observatory (SAO, Russia) $6 \mathrm{~m}$ and Observatoire de Haute Provence (OHP, France) $1.93 \mathrm{~m}$ telescopes. Later on, spectra were obtained for more 83 BIG objects in Sloan Digital Sky Survey (SDSS). We have extracted and studied these spectra, classified 
them and measured spectral features. Diagnostic diagrams have been built to distinguish starbursts (SB), LINERs and Seyfert galaxies. Cross-correlations were made for these objects with multiwavelength (MW) catalogues and their physical properties were studied. Among these 83 objects, 55 HII, 8 Seyfert galaxies, 2 LINERs, 4 other AGN, 6 composite spectrum objects, and 8 other emission-line galaxies have been revealed. Three of these objects are Ultra-Luminous InfraRed Galaxies (ULIRG). We give in Figure 4 the dependence of $\mathrm{L}_{i r}$ and $\mathrm{L}_{f i r}$ vs. redshift.
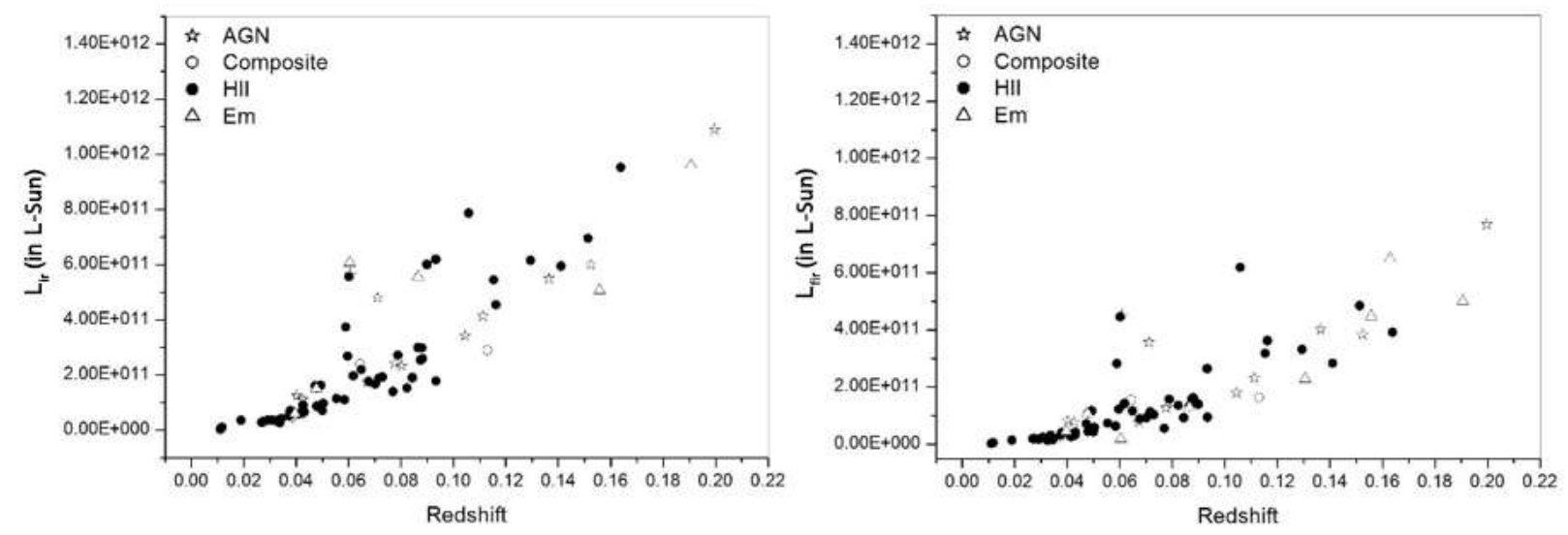

Figure 4. The dependence of $\mathrm{L}_{i r}$ and $\mathrm{L}_{f i r}\left(\right.$ in $\left.\mathrm{L}_{\odot}\right)$ vs. redshift

\section{Observations and study of Byurakan-IRAS Galaxies were summa- rized (Mikayelyan et al. 2019a)}

A general analysis of optical spectroscopic data on 257 Byurakan-IRAS Galaxies (BIG objects) obtained with the BAO $2.6 \mathrm{~m}$, SAO $6 \mathrm{~m}$, OHP $1.93 \mathrm{~m}$ telescopes and taken from SDSS spectroscopic database was carried out. 149 star-formation regions (SB) galaxies, 42 galaxies with active nuclei (AGN), and 28 galaxies with a composite spectrum were identified. The spectra of 21 galaxies show signs of emission, but without the possibility of more precise determination of their activity type (we assign them as Em), 13 galaxies appear to have star formation rates that do not exceed normal (we assign them as HII), and 3 are absorption galaxies (we assign them as Abs). We give in Table 1 the the distribution of 257 BIG objects by activity types.

Table 1. The distribution of 257 BIG objects by activity types.

\begin{tabular}{|c|c|c|c|c|c|}
\hline Activity type & Number of objects & $\%$ & Activity type & Number of objects & $\%$ \\
\hline HII & 149 & 58.0 & AGN & 13 & 5.1 \\
Composite & 22 & 8.6 & Em & 21 & 8.2 \\
HII / LINER & 4 & 1.5 & Norm & 13 & 5.1 \\
HII / Sy & 2 & 0.8 & Abs & 3 & 1.1 \\
LINER & 12 & 4.6 & Unknown & 1 & 0.4 \\
Sy & 17 & 6.6 & All & $\mathbf{2 5 7}$ & $\mathbf{1 0 0 . 0}$ \\
\hline
\end{tabular}

\section{Discovery of new bright ULIRGs from the IRAS PSC/FSC Combined Catalogue (Mikayelyan et al. 2018)}

High-luminosity IR galaxies (LIRGs, ULIRGs, and HLIRGs) are important for studies related to star-formation processes in the early Universe, as their luminosity allows to detect them at large distances. High IR indicates active star-formation and often starburst processes, which is typical to HII (starburst, SB) and AGN. An interesting question is whether the starburst triggers AGN or vice versa 
or there is no direct impact. Considering that very often such objects manifest double and multiple structure, it is also interesting to investigate the interrelationship between the SB, nuclear activity and interactions or merging. We have analyzed the IRAS PSC/FSC Combined Catalogue for search for new bright ULIRGs. By means of the SDSS DR14 data, namely redshifts for those objects having spectroscopic data, we have calculated the IR luminosities and have found 114 very high-luminosity IR galaxies; 107 ULIRGs and 7 HLIRGs. Among them, 48 new ULIRGs and 7 new HLIRGs have been discovered. These objects have been studied by SDSS color-color, luminosity-redshift and other diagrams. Further studies will include the content of the sample for activity types and other available data. We give in Figure 5 SDSS u-g vs. g-r color-color diagram and in Figure 6, the IR luminosity vs. redshift for 114 newly revealed ULIRGs and HLIRGs.
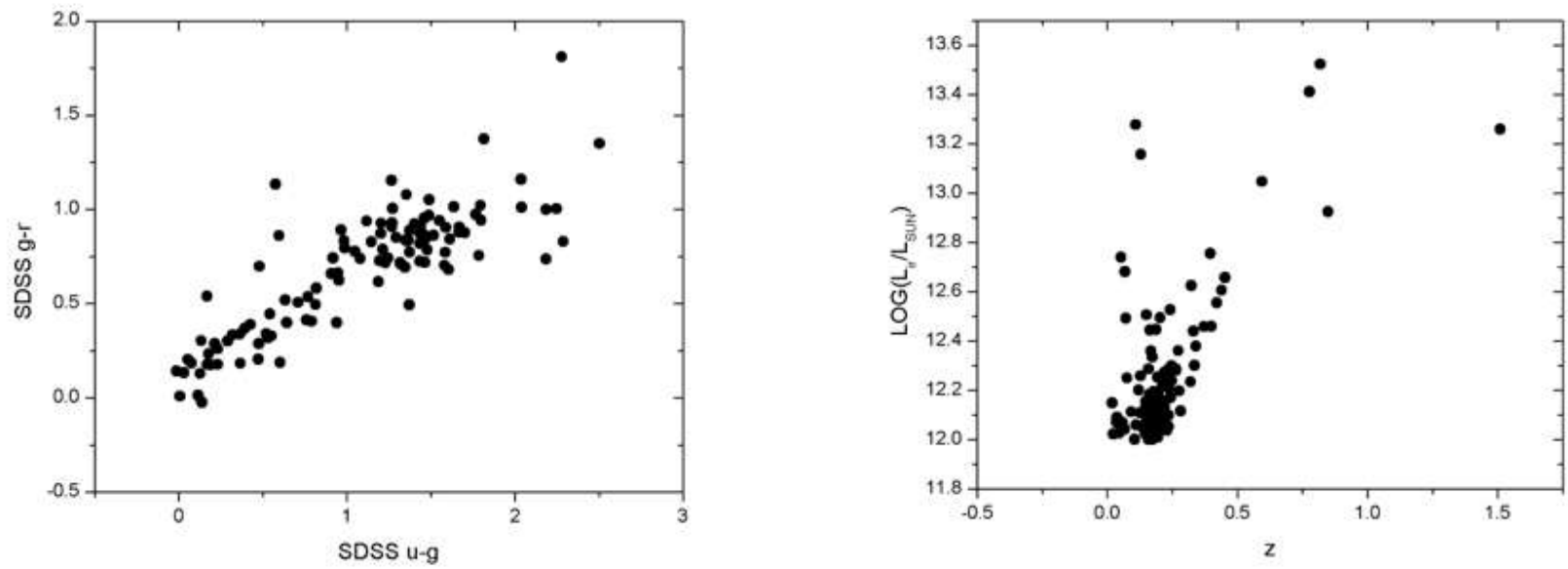

Figure 5. SDSS color-color diagram for 114 newly revealed ULIRGs and HLIRGs.

Figure 6. IR luminosity vs. redshift for 114 newly revealed ULIRGs and HLIRGs.

\section{Spectral Classification of ULIRGs from IRAS PSC/FSC Combined Catalogue (Mikayelyan et al. 2019b)}

High-luminosity IR galaxies (LIRGs, ULIRGs, and HLIRGs) are important for studies related to star-formation processes in the early Universe, as their luminosity allows to detect them at large distances. High IR indicates active star-formation and often starburst processes, which is typical to HII (starburst, SB). In many cases high IR indicates an Active Galactic Nuclei (AGN). An interesting question is whether the starburst triggers AGN or vice versa or there is no direct impact. Considering that very often such objects manifest double and multiple structure, it is also interesting to investigate the interrelationship between the SB, nuclear activity and interactions or merging. As a result of crosscorrelation of the IRAS PSC/FSC Combined catalogue with SDSS DR14, 114 ULIRGs were separated and classified by the activity types. 1 BLL, 2 quasars, 29 Seyferts of types 1.0-1.8, 5 Seyferts of type 2, 14 LINERS, 36 HII, 14 objects with a composite spectrum (Composite) were identified. Among the type 1 Seyfert galaxies there are many objects with narrow lines. We give in Figure 7 typical examples of SDSS spectra for each activity type.

\section{Radio variable sources at $1400 \mathrm{MHz}$ and their optical variability (Abrahamyan et al. 2018b)}

In the present study we have cross-correlated NVSS and FIRST radio catalogues having radio flux measurements at the same $1.4 \mathrm{GHz}$ frequency. This way we benefit from repeated observations from both catalogues, as they give more accurate positions and fluxes and more important, reveal large differences between the two measured fluxes, thus allowing to establish radio variability. As 


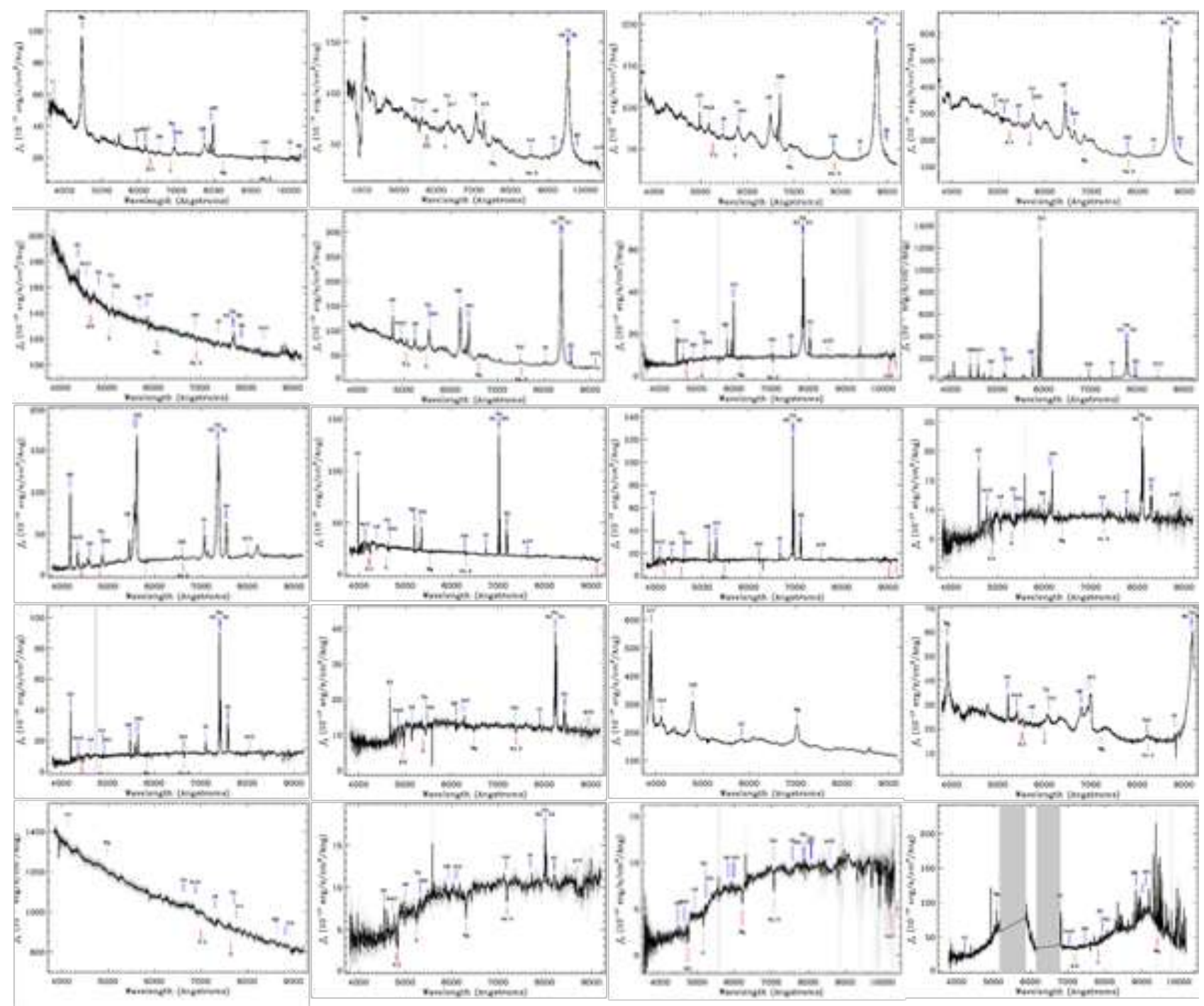

Figure 7. Typical examples for each activity type (from left to right and from top to bottom): S1.0, NLS1.0, S1.2, NLS1.2, S1.5, NLS1.5, S1.8, S2.0, LINER, HII, S1.9/HII, S1.9/LINER, LINER/HII, NLAGN, QSO, NLQSO, BL Lac, Em, Abs, Unkn.

a result, 79,382 radio variables have been revealed, including 6301 with flux differences at $1.4 \mathrm{GHz}$ larger than 15 mJy, 1917 with flux differences $>45 \mathrm{mJy}$ and 260 with flux differences $>200 \mathrm{mJy}$. By using a special technique (Mickaelian \& Sinamyan 2010, Mickaelian et al. 2011), 2425 optically variable objects out of 6301 radio sources have been revealed. 2425 radio sources with both high radio and optical variability into four categories have been divided. 1206 (19\%) out of 6301 radio sources have activity types from available catalogues and $619(25.5 \%)$ out of 2425 radio sources with at the same time radio and optical variability have activity types from available catalogues. In addition, 279 radio sources out of 2425 have high variability in optical range. We have established their activity types when available. The IR fluxes and colors for the 6301 variable radio sources have been studied. Color-color diagrams show that most of the "unknown" sources are galaxies. The activity types for $110(42 \%)$ out of 260 extremely high variable radio sources also have been retrieved. We give in Table 2 the distribution of the activity types of 6301 radio sources having radio variability and in Table 3 , the distribution of the activity types of 2425 radio sources having both radio and optical variability. 
Table 2. Activity types of 6301 radio sources having radio variability.

\begin{tabular}{|c|c|c|}
\hline No. & Activity type & Numbers \\
\hline 1 & Blazar (BZB, BZG, BZQ, BZU) & 308 \\
\hline 2 & QSO & 639 \\
\hline 3 & Sy 1.0 / Sy 1 & 19 \\
\hline 4 & Sy 1.2 & 2 \\
\hline 5 & Sy 1.5 & 6 \\
\hline 6 & Sy 1.9 & 2 \\
\hline 7 & Sy 2.0 / Sy 2 & 9 \\
\hline 8 & AGN & 97 \\
\hline 9 & Starburst & 1 \\
\hline 10 & FSS (Flat-Spectrum radio source) & 87 \\
\hline \multirow{3}{*}{11} & USS (Ultra-Steep-Spectrum radio source) & 36 \\
\hline & Known (total) & $1206(19 \%)$ \\
\hline & Unknown & $5095(81 \%)$ \\
\hline \multicolumn{2}{|r|}{ Total } & $6301(100 \%)$ \\
\hline
\end{tabular}

Table 3. Activity types of 2425 radio sources having both radio and optical variability.

\begin{tabular}{|c|l|c|}
\hline No. & Activity type & Numbers \\
\hline 1 & Blazar (BZB, BZG, BZQ, BZU) & 176 \\
2 & QSO & 333 \\
3 & Sy 1.0 / Sy 1 & 9 \\
4 & Sy 1.5 & 6 \\
5 & Sy 2.0 / Sy 2 & 5 \\
6 & AGN & 45 \\
7 & FSS (Flat-Spectrum radio source) & 41 \\
8 & USS (Ultra-Steep-Spectrum radio source) & 4 \\
\hline \multicolumn{2}{|c|}{ Total } & $\mathbf{1 8 0 6}(\mathbf{7 4 . 5} \%)$ \\
\hline & Known (total) & $\mathbf{2 4 2 5}(\mathbf{1 0 0} \%)$ \\
\hline \multicolumn{2}{|c}{} \\
\hline
\end{tabular}

\section{Classification of BZCAT objects having uncertain types (Abrahamyan et al. 2019a)}

Classification of BZCAT objects having uncertain types was aimed at understanding some optical properties of blazars having uncertain types (BZU) in BZCAT Catalogue v5. Cross-correlation with SDSS revealed $43 \mathrm{BZU}$ objects that have spectra in SDSS out of the total $227 \mathrm{BZU}$ ones. We have carried out spectral re-classification for these 43 blazar candidates for activity types. As a result, 37 (86\%) objects out of 43 changed their previous type. We give in Table 4 the new classification of former BZU objects and in Table 5, spectral classification of these objects for activity types using SDSS spectra.

Table 4. New classification of BZU objects.

\begin{tabular}{|c|c|c|c|}
\hline No. & Old & New & Numbers \\
\hline 1 & BZU & BZB & $1(2 \%)$ \\
2 & BZU & BZG & $14(33 \%)$ \\
3 & BZU & BZQ & $22(51 \%)$ \\
4 & BZU & BZU & $6(14 \%)$ \\
\hline \multicolumn{3}{|c|}{ All } & $\mathbf{4 3 ( 1 0 0 \% )}$ \\
\hline
\end{tabular}


Table 5. Spectral classification using SDSS spectra.

\begin{tabular}{|c|c|c|}
\hline No. & Activity Type & Numbers \\
\hline 1 & Abs & 1 \\
2 & BLL & 1 \\
3 & Em & 4 \\
4 & LINER & 2 \\
5 & NLQSO & 1 \\
6 & QSO & 17 \\
7 & QSO 1.2 & 3 \\
8 & QSO 1.5 & 1 \\
9 & Sy 1.2 & 1 \\
10 & Sy 1.5 & 2 \\
11 & Sy 1.8 & 1 \\
12 & Sy 2.0 & 3 \\
13 & Unknown & 6 \\
\hline \multicolumn{2}{|c|}{ Total } & $\mathbf{4 3}$ \\
\hline
\end{tabular}

\section{Optical variability of blazars (Abrahamyan et al. 2019b)}

The analysis of blazars' parameters from BZCAT leads to a conclusion that they do not have the same properties. The preliminary criterion to include an object in the catalog was the strong radio emission; however, two type of radio sources were selected: BL Lacertae (BLL) objects and Flat Spectrum Radio Quasars (FSRQ). As a number of properties are typical of blazars (strong radio emission, optical variability, continuum optical spectra, polarization, high luminosity, etc.), using the optical data, we investigate them to clarify which property plays the most significant role in their classification as blazars. We found that $60 \%$ of blazars have optical variability. We use a technique developed based on POSS1 and POSS2 photometry and group the variability into extreme, strong, medium, and low classes. In the optical range, 51 blazars have powerful variability (extreme variables), and 126 are high variables. In addition, $63 \%$ of blazars have detected radiation in X-ray and $28 \%$ have detected radiation in gamma rays. We give the average statistical characteristics of blazars based on our analysis and calculations. Here we give in Figure 8 absolute magnitude vs. redshift for BZCAT blazars.
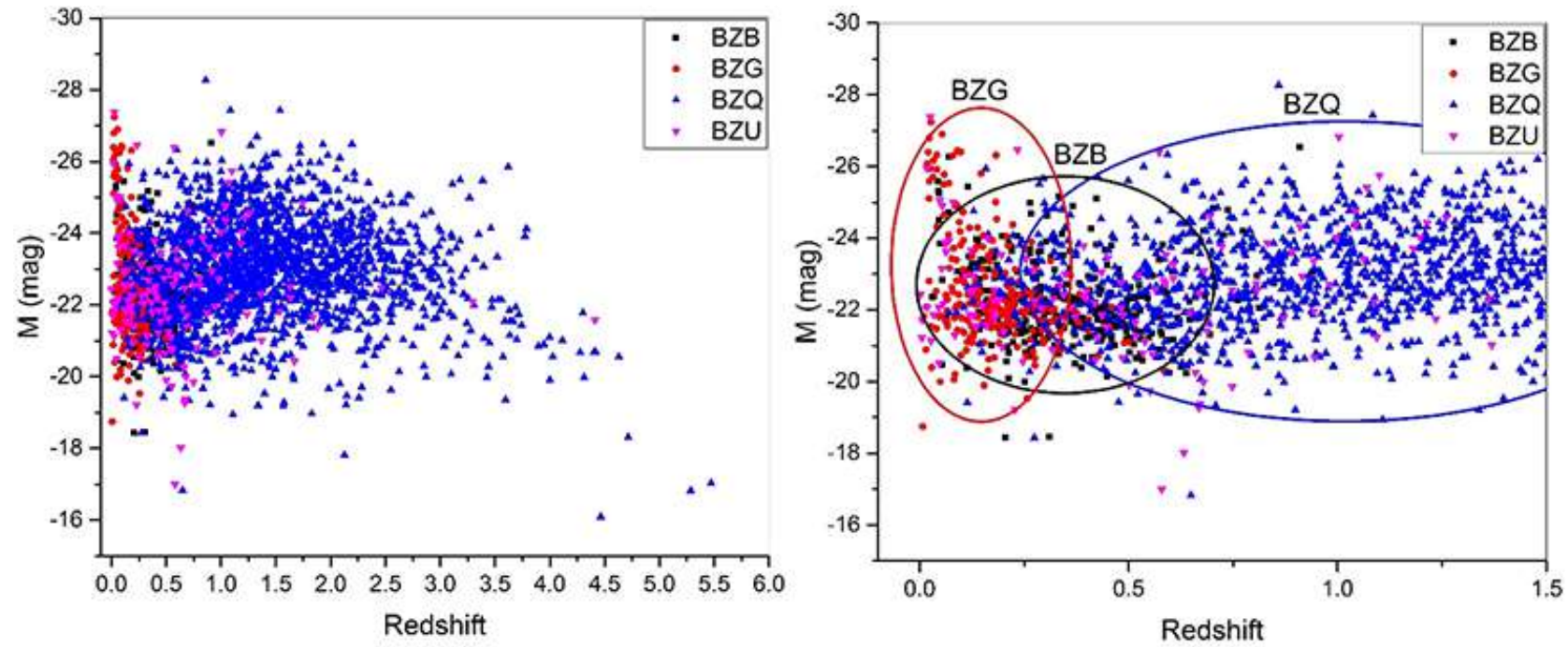

Figure 8. Absolute magnitude vs. redshift for BZCAT blazars. 


\section{Summary}

A number of interesting results have been obtained in the field of multiwavelength studies of active galaxies, including X-ray, IR and radio sources identified with AGN and Starbursts. Among the important results one could mention:

- Study of SDSS spectra of 371 optical identifications of HRC/BHRC Sample ROSAT X-ray sources (Paronyan et al. 2018, 2019a,b). These sources were also identified in other wavelength ranges and some of their parameters were calculated.

- The UV properties of 1152 Markarian galaxies were investigated based on GALEX data (Mickaelian et al. 2018a). Using our classification for activity types for 779 Markarian galaxies based on SDSS spectroscopy, we have investigated these objects on the GALEX, 2MASS and WISE color-magnitude and color-color diagrams by the location of objects of different activity types and have revealed a number of concentrations.

- Abundance and Star Formation Determinations in more than 700 Markarian galaxies from SDSS Spectra (Gyulzadyan et al. 2018). We have measured their line fluxes and derived the $\mathrm{O}$ and $\mathrm{N}$ abundances using recent calibrations. The behavior of the $[\mathrm{N} / \mathrm{H}]$ ratio in under-abundant regions gives strong support to a partially primary origin of nitrogen.

- Revised optical classification of objects classified as "LINERs" in the Catalogue Véron-Cetty \& Véron 13th edition (Abrahamyan et al. 2018a), where 926 LINERs are included. We found 176 objects having spectra in SDSS DR14. The re-classification of these objects led to $54 \%$ of them to change their activity type classifications.

- Study and classification of SDSS spectra for Byurakan-IRAS Galaxies (Mickaelian et al. 2018b). To the previously observed 172 BIG objects (with BAO, SAO and OHP telescopes) 83 BIG objects were added having spectra in SDSS. Among them, 55 HII, 8 Seyfert galaxies, 2 LINERs, 4 other AGN, 6 composite spectrum objects, and 8 other emission-line galaxies have been revealed. Three of these objects are ULIRGs. The observations and study of 257 Byurakan-IRAS Galaxies were summarized in Mikayelyan et al. (2019a).

- Discovery of new bright ULIRGs from the IRAS PSC/FSC Combined Catalogue (Mikayelyan et al. 2018). By means of the SDSS DR14 data, namely redshifts for those objects having spectroscopy, we have calculated the IR luminosities and have found 114 very high-luminosity IR galaxies; 107 ULIRGs and 7 HLIRGs. Among them, 48 new ULIRGs and 7 new HLIRGs have been discovered. Spectral classification of these ULIRGs were carried out (Mikayelyan et al. 2019b). 1 BLL, 2 quasars, 29 Seyferts of types 1.0-1.8 (including narrow-line), 5 Seyferts of type 2, 14 LINERS, 36 HII, 14 objects with a composite spectrum (Composite) were identified.

- Radio variable sources at $1400 \mathrm{MHz}$ were studied and their optical variability was revealed (Abrahamyan et al. 2018b). As a result, 79,382 radio variables have been revealed, including 6301 with flux differences at $1.4 \mathrm{GHz}$ larger than $15 \mathrm{mJy}$. By using a special technique (Mickaelian \& Sinamyan 2010, Mickaelian et al. 2011), 2425 optically variable objects out of 6301 radio sources have been revealed.

- Classification of BZCAT objects having uncertain types was carried out (Abrahamyan et al. 2019a). 43 BZU objects were found to have SDSS spectra and as a result, 37 (86\%) objects changed their previous type.

- Optical variability of blazars was studied (Abrahamyan et al. 2019b). We found that $60 \%$ of blazars have optical variability. In the optical range, 51 blazars have powerful variability (extreme variables), and 126 are high variables. In addition, $63 \%$ of blazars have detected radiation in $\mathrm{X}$-ray and $28 \%$ have detected radiation in gamma rays. 


\section{List of Used Abbreviations}

2MASS - 2 Micron All-Sky Survey

AGN - Active Galactic Nucleus

BAO - Byurakan Astrophysical Observatory (Armenia)

BIG - Byurakan-IRAS Galaxy

BLL - BL Lacertae object

BZCAT - Roma Blazars Catalogue

BZG - BZCAT Galaxy

BZQ - BZCAT Quasar

BZU - BZCAT Uncertain type object

DR - Data Release

FIRST - Faint Images of the Radio Sky at Twenty-centimeters

FSC - Faint Source Catalogue

FSRQ - Flat Spectrum Radio Quasars

FSS - Flat Spectrum radio Source

FUV - Far UltraViolet

GALEX - Galaxy Explorer

HBRC - Hamburg-Byurakan-ROSAT Catalogue

HLIRG - Hyper-Luminous InfraRed Galaxy

HRC - Hamburg-ROSAT Catalogue

IFS - Integral Field Spectroscopy

IRAS - InfraRed Astronomical Satellite

LINER - Low Ionization Nuclear Emission-line Region

LIRG - Luminous InfraRed Galaxy

NUV - Near UltraViolet

NVSS - NRAO/VLA Sky Survey

OHP - Observatoire de Haute Provence (France)

POSS1 - Palomar Observatory Sky Survey 1

POSS2 - Palomar Observatory Sky Survey 2

PSC - Point Source Catalogue

SAO - Special Astrophysical Observatory (Russia)

SB - StarBurst galaxy

SDSS - Sloan Digital Sky Survey

SED - Spectral Energy Distributions

SFR - Star Formation Rate

ULIRG - Ultra-Luminous InfraRed Galaxy

USS - Ultra Steep Spectrum radio Source

WISE - Wide-field Infrared Survey Explorer 


\section{References}

Abrahamyan H. V., Mickaelian A. M., Mikayelyan G. A., Paronyan G. M., 2018a, ComBAO, 65, 1

Abrahamyan H. V., Mickaelian A. M., Paronyan G. M., Mikayelyan G. A., Gyulzadyan M. V., 2018b, Astronomy \& Computing, 25, 176

Abrahamyan H. V., Mickaelian A. M., Paronyan G. M., Mikayelyan G. A., Gyulzadyan M. V., 2019a, ComBAO, 66, 1

Abrahamyan H. V., Mickaelian A. M., Paronyan G. M., Mikayelyan G. A., 2019b, AN, 340, 437

Aguado D. S., Romina A., Andrés A., et al. 2019, ApJS, 240, id. 23

Bianchi L., Herald J., Efremova B., et al. 2011, Ap\&SS, 335, 161

Bird A. J., Bazzano A., Bassani L., et al. 2010, ApJS, 186, 1

Condon J., Cotton W., Greisen E., et al. 1998, AJ, 115, 1693

Gyulzadyan M. V., Mickaelian A. M., Abrahamyan H. H., Mikayelyan G. A., Paronyan G. M., 2018, ComBAO, 65, 392

Helfand D. J., White R., Becker R. H., 2015, ApJ, 801, id. 26

IRAS 1988, Joint IRAS Science W.G., IRAS Catalog of Point Sources, Version 2.0

Massaro E., Alessandro M., Cristina L., et al. 2015, Ap\&SS, 357, id. 75

Mickaelian A. M., 2017, ComBAO, 64, 15

Mickaelian A. M., Sinamyan P. K., 2010, MNRAS, 407, 681

Mickaelian A. M., Hovhannisyan L. R., Engels D., Hagen H.-J., Voges W., 2006, A\&A, 449, 425

Mickaelian A. M., Mikayelyan G. A., Sinamyan P. K., 2011, MNRAS, 415, 1061

Mickaelian A. M., Gyulzadyan M. V., Abrahamyan H. V., Paronyan G. M., Mikayelyan G. A., 2017, "Non-Stable Universe: Energetic Resources, Activity Phenomena, and Evolutionary Processes", Proc. Intl. Symp. dedicated to 70th anniv. of Byurakan Astrophysical Observatory (BAO), held in Yerevan and Byurakan, Armenia 19-23 September 2016. Eds. A. M. Mickaelian, H. A. Harutyunian, E. H. Nikoghosyan. ASP Conf. Ser., 511, 149

Mickaelian A. M., Abrahamyan H. V., Gyulzadyan M. V., Paronyan G. M., Mikayelyan G. A., 2018a, Ap\&SS, 363, id. 237

Mickaelian A. M., Harutyunyan G. S., Sarkissian A., 2018b, Astronomy Letters, 44, 351

Mikayelyan G. A., Mickaelian A. M., Abrahamyan H. V., Paronyan G. M., 2018, ComBAO, 65, 13

Mikayelyan G. A., Mickaelian A. M., Abrahamyan H. V., Paronyan G. M., Gyulzadyan M. V., 2019a, ComBAO, 66, 31

Mikayelyan G. A., Mickaelian A. M., Abrahamyan H. V., Paronyan G. M., Gyulzadyan M. V., 2019b, Ap, 62, 503

Moshir M., Copan G., Conrow T., et al. 1989, IRAS Faint Source Catalog, Version 2.0, NASA

Paronyan G. M., Mickaelian A. M., Abrahamyan H. V., Mikayelyan G. A., 2018, ComBAO, 65, 412

Paronyan G. M., Mickaelian A. M., Harutyunyan G. S., Abrahamyan H. V., Mikayelyan G. A., 2019a, Ap, 62, 147 
Paronyan G. M., Mickaelian A. M., Abrahamyan H. V., Mikayelyan G. A., 2019b, Ap, in press

Véron-Cetty M. P., Véron P., 2010, A\&A, 518, A10

Voges W., Aschenbach B., Boller T., et al. 1999, A\&A, 349, 389

Voges W., Aschenbach B., Boller T., et al. 2000, MPE, Garching. The ROSAT Faint Source Catalogue

Zickgraf F.-J., Engels D., Hagen H.-J., Reimers D., Voges W., 2003, A\&A, 406, 535 